\title{
EL APRENDIZAJE DEL EMPRENDIMIENTO TECNOLÓGICO E INNOVACIÓN DESDE UN AMBIENTE UNIVERSITARIO CON IMPACTO A LA SOCIEDAD
}

\author{
The Learning of Technological Entrepreneurship and Innovation \\ from a University Environment with Impact to Society
}

\author{
SANDRA HERNÁNDEZ LóPEZ
}

Universidad Autónoma de Querétaro, México

\section{KEY WORDS}

\section{Learning}

Technology

Entrepreneurship and

Innovation

University Environment

Social impact

\section{ABSTRACT}

In Mexico before September 30, 2019, public universities, was to train employees generating unemployment in graduate students. By establishing a program of technological entrepreneurship and innovation, will it comply with the LGE? The general objective of carrying out a study of the impact of entrepreneurship received during their training as engineers in mechatronic terminal line automation of active students and graduates 2015-2018, this will allow to develop an entrepreneurship program. Methodology is quantitative, phenomenological and ethnographic, in the UAQ in the FI applying surveys. Results are optional and not curricular. Conclusion The proposed entrepreneurship study program complies with the LGE.
PALABRAS CLAVE

Aprendizaje

Emprendimiento tecnológico e innovación

Ambiente universitario

Impacto social

\section{RESUMEN}

En México antes del 30 de septiembre de 2019 las Universidades públicas, era formar empleados generando desempleo en estudiantes egresados. ¿Estableciendo un programa de emprendimiento tecnológico e innovación, cumplirá con la LGE? El objetivo general realizar un estudio del impacto del emprendimiento recibido durante su formación como ingenieros en automatización línea terminal mecatrónica de estudiantes activos y egresados 2015-2018, esto permitirá desarrollar un programa de emprendimiento. Metodología es cuantitativa, fenomenológica y etnográfica, en la UAQ en la FI aplicando encuestas. Resultados son optativas y no curriculares. Conclusión el programa de estudio de emprendimiento propuesto cumple con la LGE. 


\section{Introducción}

$\mathrm{E}$ n México el desempleo de los estudiantes egresados se incrementado año con año como se apreciar en la gráfica 1.1 Tasa de desempleo promedio por año de educación medio y superior emitido por el Instituto de estadística y geografía (INEGI, 2019), refleja la preocupación de las autoridades motivo por lo cual nacen instituciones gubernamentales para fomentar el emprendimiento por medio de la Secretaria de Economía entre ellas el Instituto Nacional del Emprendedor (INADEM, 2018) su objetivo es apoyar a las incubadoras de empresas públicas y privadas del país para fomentar el emprendimiento, no teniendo resultados favorables en su totalidad.

Gráfica 1. Tasa de desempleo promedio por año de educación medio y superior.

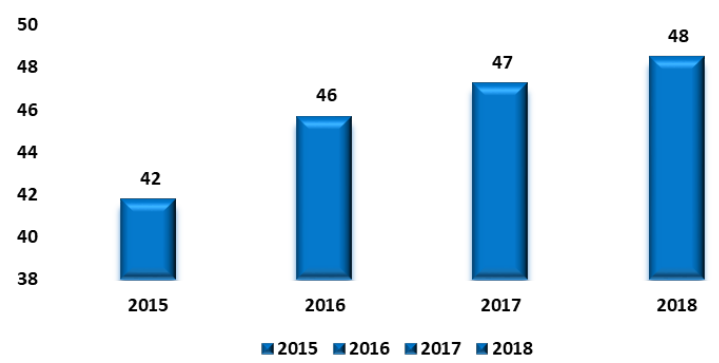

Fuente. Elaboración propia. (INEGI, 2019)

La Organización para la Cooperación y el Desarrollo Económicos (OCDE) recomendó a las universidades mexicanas fortalecer las estrategias de fomento emprendedor, mecanismos para apoyar a los emprendedores para la creación de empresas, las universidades implementar programas que promueven el espíritu emprendedor y temas de financiamiento, la cultura emprendedora de estudiantes, docentes e investigadores fortaleciendo vínculos. Las universidades deben realizar políticas y estructuras para ser un apoyo para las incubadoras universidad-empresa para la incubadora. (Martín Carbajal \& Gutiérrez Hernández, 2018)

En algunas universidades este tema no se aborde en todas las licenciaturas, solo las económicas administrativas por su naturaleza, hoy es importante instituirlas en los programas académicos en todas las licenciaturas, debido a la falta de empleo es necesario generar una cultura emprendedora. ( Hernández Rodríguez \& Arano Chávez, 2015)

La Asociación Nacional de Universidades e Instituciones de Educación Superior (ANUIES) menciona que las empresas se aprovechan ofreciendo menos sueldo a personal altamente calificado, algunos otros culpan al sistema educativo debido que el estudiante obtiene buenos conocimientos y poca experiencia práctica. ( Hernández Rodríguez \& Arano Chávez, 2015)

Se sustenta esta investigación con el siguiente cuestionamiento ¿Estableciendo un programa de emprendimiento tecnológico e innovación, cumplirá con la LGE?

El objetivo general realizar un estudio del impacto del emprendimiento recibido durante su formación como ingenieros en automatización línea terminal mecatrónica de estudiantes activos y egresados 2015-2018, esto permitirá desarrollar un programa de emprendimiento.

Metodología es cuantitativa, fenomenológica y etnográfica, en la Universidad Autónoma de Querétaro en la Facultad de Ingeniería línea terminal mecatrónica de activos y egresados del 2015 al 2018, por medio del cuestionario.

Hipótesis cierta si se realiza un estudio del aprendizaje emprendimiento tecnológico e innovación desde un ambiente universitario con impacto social, recibido durante su formación como ingenieros en automatización línea terminal mecatrónica de estudiantes activos y egresados 2015-2018, entonces se logrará desarrollar un programa de estudio de emprendimiento.

Hipótesis nula si se realiza un estudio del aprendizaje emprendimiento tecnológico e innovación desde un ambiente universitario con impacto social, recibido durante su formación como ingenieros en automatización línea terminal mecatrónica de estudiantes activos y egresados 2015-2018, entonces no se logrará desarrollar un programa de estudio de emprendimiento.

Variable dependiente la ausencia del ecosistema emprendedor en los programas de estudio, con asignaturas curriculares de emprendimiento.

Variables independiente ausencia de visión emprendedora de los egresados, los programas 
de estudio sin actualización y la empatía para dar un vistazo a un mundo globalizado.

Es viable por cooperación de la Coordinación de ingeniería en automatización línea terminal mecatrónica de la información necesaria para realizarla investigación, el acercamiento a los egresados y los jóvenes activos en la Facultad de ingeniería.

Pertinencia las condiciones del país de forma política, económica y social es adecuada para desarrollar la investigación en la Universidad Autónoma de Querétaro.

\section{Marco teórico}

La importancia del ecosistema emprendedor en los programas de estudio de la Facultad de ingeniería en automatización, línea terminal mecatrónica de la Universidad Autónoma de Querétaro, para futuras generaciones y ser ejemplo para otras Facultades que no cuentan con conocimientos económico administrativo, logrando el aprendizaje del emprendimiento tecnológico e innovación desde un ambiente universitario con impacto a la sociedad.

En los siguientes párrafos se localizarán diferentes autores que han estudiado la experiencia de agregar a sus programas de estudio el emprendimiento.

En el artículo publicado por la revista Didáctica y Educación "La innovación y el emprendimiento: necesidades en la educación superior" Álvarez Perdomo, Andrade Alcívar, Bravo Salvatierra, Rodríguez Martínez, \& Govea Vilcacundo, 2016 mencionan:

El enfoque innovador y emprendimiento en algunas universidades de Latinoamérica, en el desarrollo de las investigaciones científicas es bajo, son poco sensibilizados en docentes, estudiantes y la comunidad universitaria considerándolos no importantes para su crecimiento y fortalecimiento de los centros de investigación.

Las universidades deben generar espacios que incentiven el desarrollo de la innovación y el emprendimiento en centros de investigación, parques tecnológicos, laboratorios, etc permitiendo proyectos en conjunto de otras áreas o facultades que permitan el conocimiento trasversal.
Es una transformación organizacional de las universidades que no es de la noche a la mañana, es de forma paulatina y se necesita capacitar a los docentes, estudiantes y comunidad universitaria para generar el interés, principalmente en las asignaturas que han incorporado en el programa académico.

Una forma de incorporar estos temas de innovación y emprendimiento es agrupar a las diferentes universidades para que se determinen las estrategias de acción que permitan fortalecer el ecosistema emprendedor en universidades de Latinoamérica. (Álvarez Perdomo, Andrade Alcívar, Bravo Salvatierra, Rodríguez Martínez, \& Govea Vilcacundo, 2016)

Cavero y Ruíz argumentan en su libro "Educación para la innovación y el emprendimiento una educación para el futuro". Es necesario considerar mentores para colegios y universitarios que tengan experiencia en el emprendimiento como son empresarios y gente que está involucrada en una organización, se le permita dar clases y en los cuerpos colegiados para la definición de programas académicos. (Cavero Clerencia \& Ruíz Clerencia, 2017)

Vásquez César en su artículo publicado en la revista estudios de la Gestión: revista internacional de administración del año 2017 titulado "Educación para el emprendimiento en la universidad" aportación es la siguiente los docentes deben inculcar $y$ fomentar el emprendimiento e innovación en los estudiantes de forma analítica y planificada con una actitud y sensibilización al resolviendo problemas del país.

El docente de debe estar de forma permanente al cuidado de los estudiantes emprendedores para resolver dudas $y$ encausarlos, creando habilidades y actitudes emprendedoras e innovadoras en ellos, incluso aprendan de ellos mismos, implementado conocimiento cognitivo y didáctico adecuado para la generación de ideas reales para problemas que la sociedad demande. (Vásquez , 2017)

"Enseñanza del emprendimiento en la educación superior: ¿Metodología o modelo?" (Saldarriaga Salaza \& Guzmán González, 2018 de la Universidad de Viña del Mar, Chile encuentra los siguientes hallazgos: 
1. Solo algunas universidades cuentan con estudios de los métodos, metodologías y guías para crear un modelo de enseñanza en emprendimiento propio.

2. Estos métodos, metrologías y guías resultando un modelo de aprendizaje en emprendimiento son aplicados en diferentes carreras.

3. Se implementaron desde el diseño curricular, el objetivo, orientación práctica en la generación de ideas emprendedoras en un ambiente de aprendizaje y evaluación del modelo.

4. Se capacitaron a los docentes para trabajar con los estudiantes en el emprendimiento en los temas de argumentación, reflexión e interpretación y se evalúa la metodología.

5. La finalidad de la implementación del emprendimiento en las materias curriculares crea su propia empresa.

6. Se les prepara para la toma de decisiones acertadas por medio de acercarlos a un mundo real, conociendo los problemas del mundo emprendedor de tal manera que no abandonen y que sigan buscando oportunidades de crecimiento.

7. En algunos países se han implementado en los programas de estudio a fin de promover el emprendimiento en las universidades, esto género que para cumplir con esta política se tomen modelos existes y no crean su propio modelo ya que cada universidad tiene su propio sistema de enseñanza.

8. Estas universidades que implementaron en sus programas con la finalidad de bajar recursos para llevar acabo los emprendimientos locales, regionales, nacionales e internacionales.

9. Se perdió el objetivo de emprendimiento en conjunto, es decir la idea se convierte en negocio, sin perder de vista la formación del estudiante con las habilidades y actitudes para emprender, crecer y permanecer en el negocio.

10. Se enseña en el programa de estudio por cumplir un requisito de curricular. (Saldarriaga Salaza \& Guzmán González, 2018)
Resumiendo, el aprendizaje del emprendimiento tecnológico e innovación desde un ambiente universitario con impacto a la sociedad, ha estado evolucionado paulatinamente de acuerdo a las necesidades como lo es el desempleo generado en los estudiantes egresados y el poco apoyo de los gobiernos a las universidades, la necesidad apremia para resolver problemas que presenta la sociedad en sus diferentes áreas y una en común el desempleo.

Para esto es necesario capacitar a los docentes, personal administrativo y los coordinadores de licenciaturas para implementar en los programas académicos el tema del emprendimiento e innovación, estableciendo estrategias, metodologías $y$ métodos que permitan crear habilidades $y$ actitudes emprendedoras e innovadoras para resolver problemas que tiene la sociedad.

\subsection{Marco conceptual}

Es importante destacar los conceptos que se trataron en esta investigación, todos relacionados y unidos por un ambiente universitario, por medio del aprendizaje del emprendimiento tecnológico e innovador con impacto social. Figura 1 Marco conceptual.

Imagen 1. Marco conceptual

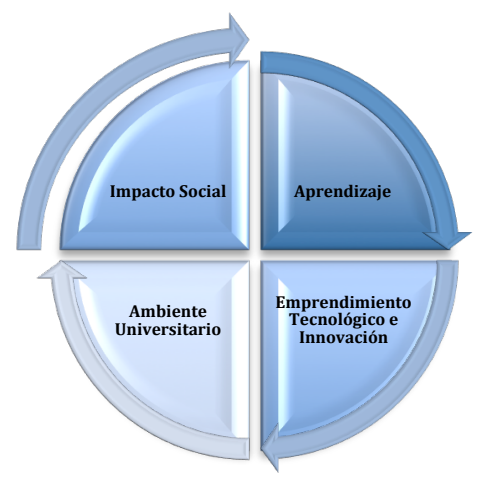

Fuente: Elaboración propia, 2019.

En el "Impacto social y académico del programa interacción social y desarrollo ciudadano en tres localidades del departamento del atlántico" de Angélica Orozco Idarraga en el año 2014 define del Evaluación del impacto social (EIS): 
...comprende los procesos de análisis, seguimiento y gestión de las consecuencias sociales, voluntarias e involuntarias, tanto positivas como negativas, de las intervenciones planeadas (políticas, programas, planes, proyectos), así como cualquier proceso de cambio social, invocado por dichas intervenciones. (Orozco Idárraga, 2014, p. 478)

Esta definición permite conocer los beneficios la evaluación del impacto social no es solo planeada en los programas, proyectos o plan tiene efectos colaterales que suman o restan en la sociedad. En el emprendimiento aplicado a los programas de estudio tiene un beneficio para los participantes, esto implica mayor capacitación a docentes y estudiantes dispuesto a participar.

Dionicio Morales y Ruth Roux en su artículo "Estudio de impacto social: antecedentes y línea base para San Fernando, Tamaulipas" 2015 define como impacto social:

...reconoce que la significancia del impacto social varía de un lugar a otro, de un proyecto a otro, de una comunidad a otra y, hasta dentro de los grupos de una misma comunidad. (Morales Ramírez \& Roux Rodríguez, 2015, p. 112)

En el emprendimiento e innovación su impacto social será diferente en cada universidad, campus, licenciatura, ingeniería de acuerdo a la problemática a resolver y ofrecer soluciones a las necesidades de la sociedad.

"Evaluación auténtica del impacto social de procesos, proyectos y productos de investigación universitaria: un acercamiento desde los grupos de investigación" de Julia V. Escobar, Diego A. Castaño, Marilza P. Ruíz y Juan C. Restrepo en su publicación del 2016 en la revista Lasarrusta de investigación, que el impacto social:

...se sistematice la información obtenida del seguimiento a corto, mediano y largo plazo del impacto social de procesos, productos y proyectos de investigación. (Escobar Londoño, Castaño, Ruiz Ruiz, \& Restrepo Botero, p. 169)

El impacto social de emprendimiento e innovación debe planearse en los programas académicos de forma de objetivos alcanzados a corto, mediano y largo plazo en los procesos de los proyectos de investigación que se presente, pensando así tener una retroalimentación constante para su actualización.

"Enseñanza del emprendimiento en la educación superior: ¿Metodología o modelo?" artículo publicado por la revista EAN de los autores María Eugenia Saldarriaga y María Fernanda Guzmán mencionan que el aprendizaje es:

...el aprendizaje basado en proyectos para estimular y aprender el proceso del emprendedor, los estudiantes se involucran en la actividad experimental a través de proyectos para aplicar conocimientos previos en la solución de problemas. (Saldarriaga Salaza \& Guzmán González, 2018, p. 134)

El aprendizaje en el emprendimiento debe estimular el aprendizaje basado en proyectos, permitiéndole vivenciar de forma real las actividades de experimentación, donde desarrollen sus propios conocimientos basados en los adquiridos en su formación académica y obtenidos en el proyecto.

En el artículo de José Carlos Sánchez, Alezander Ward, Jenny Lizette "Educación emprendedora: Estado del arte" del año 2017 menciona que el aprendizaje:

...el aprendizaje en base a competencias, hay que tomar no solo una coyuntura que abarque conocimiento científico, sino que también promueva la creatividad en no solo pensamiento, también cultura del esfuerzo, emprendimiento, toma de decisiones, trabajo en equipo, análisis y solución de problemas, comunicación, creatividad, innovación ... (Sánchez García, Brizeida Hernández, \& Lizette Florez, 2017, p.412)

El aprendizaje en el emprendimiento debe basarse en competencias y no solo científicas, si no de habilidades blandas como es la toma de decisiones, creatividad, innovación, comunicación, trabajo en equipo una cultura de esfuerzo, aprenderán al enfrentar problemas y encontrar soluciones.

"El desarrollo de la cultura emprendedora en estudiantes universitarios para el fortalecimiento de la visión empresarial", artículo de Carlos Hernández y Raúl Arano de 2015 menciona que el ambiente debe ser de: 
La libertad es uno de los componentes para el nacimiento de un espíritu emprendedor, teniendo en cuenta que la actitud de todo emprendedor involucra comenzar, investigar, arriesgarse, las mismas sólo son posibles en un ambiente de libertad... (Hernández Rodríguez \& Arano Chávez, 2015, p. 34)

Los estudiantes deben sentirse en un ambiente universitario libre, que le permita realizar sus propias ideas con una visión de resolver alguna problemática de la sociedad, creando habilidades y actitudes en la toma decisiones y el trabajo en equipo.

"Educación para la innovación y el emprendimiento una educación para el futuro" de los autores Cavero Clerencia, José María; Ruíz Clerencia, Diego, 2017 menciona que el emprendimiento:

...es una de las competencias clave y se refiere a la capacidad del individuo para convertir ideas en acción, es decir, para llevar sus ideas a la práctica. Para completar esta definición habría que señalar que el emprendimiento debe suponer que estas acciones aporten valor, ya sea financiero, cultural o social. (Cavero Clerencia \& Ruíz Clerencia, 2017)

El emprendimiento es un conjunto de acciones que tiene la capacidad el estudiante de generar ideas y convertirlas en realidad para resolver una problemática que sea viable financieramente, cultural o social.

"Brechas para el Emprendimiento en la Alianza del Pacífico" de la organización Observatorio Estratégico de la Alianza del Pacífico Pontificia Universidad Católica de Chile del 2018 menciona:

El emprendimiento constituye un motor clave para el empleo, crecimiento y progreso de los países. (Observatorio Estratégico de la Alianza del Pacífico, 2018, p. 17)

El emprendimiento en los países de Latinoamérica es vital, para el crecimiento de las económicas de países donde existe desempleo, los organismos gubernamentales deben buscar estrategias para la implementación en el sector educativo para tener un crecimiento sustentado en capacitación en primera instancia en educación básica y cuando lleguen a educación media superior y superior tengan los conocimientos teóricos, crecido en habilidades y actitudes para emprender y generen proyectos que tengan impacto social.

México la Secretaria de Educación por medio de la Ley General de Educación agrega el 30 de septiembre del 2019 en su artículo 30 en su fracción:

...Artículo 30. Los contenidos de los planes y programas de estudio de la educación que impartan el Estado, sus organismos descentralizados y los particulares con autorización o con reconocimiento de validez oficial de estudios, de acuerdo al tipo y nivel educativo, serán, entre otros, los siguientes... XIV. La promoción del emprendimiento, el fomento de la cultura del ahorro y la educación financiera... (Cámara de diputados del $\mathrm{H}$. Congreso de la Unión, 2019)

El proceso de transición de la capacitación de docentes de primaria, secundaria, educación media superior y superior, de enseñar de forma tradicionalista donde el docente tenia por varios años estructurado su programa educativo y estar en su estado de confort, hoy tiene que capacitarse para cumplir con la ley. Para el gobierno es un gran reto para que por primera instancia es el acercamiento al docente y como consecuencia a los estudiantes, sé que se necesitara más de un sexenio se vean resultados en gran escala, considero que con un $10 \%$ de los docentes se logre capacitarlos estos contagiaran a otros, es decir se genere un ecosistema emprendedor.

La investigación trastoca la innovación por lo que se consulta en el Manual de Oslo del 2018 define innovación:

Innovation activities include all developmental, financial and commercial activities...A business innovation is a new or improved product or business process (or combination thereof)that differs significantly from the firm's previous products or business processes and that has been introduced on the market or brought into use by the firm. (OECD, 2018)

Es importante determinar el termino debido que los docentes necesitan aplicar correctamente en el proceso de enseñanza aprendizaje, como lo determina el Manual de Oslo (OECD, 
2018)menciona que todas las actividades con desarrollo financiero y comercial está involucrada la innovación y en las empresa es un producto o proceso comercial nuevo o mejorado.

De acuerdo a la Ley general de educación (Cámara de diputados del $\mathrm{H}$. Congreso de la Unión, 2019)obliga a las instituciones de educación emprendedora y financiera, así como la aplicación de la investigación, la ciencia, tecnología y la innovación como la comprensión, aplicación y el uso responsable de la misma, como se menciona enseguida:

Artículo 30. Los contenidos de los planes y programas de estudio de la educación que impartan el Estado, sus organismos descentralizados y los particulares con autorización o con reconocimiento de validez oficial de estudios, de acuerdo al tipo y nivel educativo, serán, entre otros, los siguientes ... IV. El fomento de la investigación, la ciencia, la tecnología y la innovación, así como su comprensión, aplicación y uso responsables... (Cámara de diputados del H. Congreso de la Unión, 2019)

\section{Metodología}

Metodología cuantitativa, fenomenológica y etnográfica, utilizando como herramienta cuestionario, aplicado en la ingeniería de automatización línea terminal mecatrónica del periodo 2015-2018 egresados y activos en la Universidad Autónoma de Querétaro (UAQ).

Cuantitativa es un enfoque estadístico que nos permite conocer datos duros, sobre los proyectos de investigación aportando resultados probabilísticos donde se pueden documentar y contrastar con otros, para la toma de decisiones. (Cienfuegos Velasco \& Cienfuegos Velasco, 2016)

El tipo de investigación es fenomenológica ya que se descubre y describe como ha impactado el emprendimiento en estudiantes los egresados y activos en su formación académica y la vida real, es decir, comprender este fenómeno para emprender. ( Mendieta-Izquierdo, RamírezRodríguez, \& Fuerte, 2015)

Etnográfica en el estudio educativo en un ámbito histórico, es decir, generaciones 2015 a la 2018 como impacta sus programas de estudio en ese entonces y hoy. (Gutiérrez Giraldo, Agudelo Cely, \& Orlando Caro, 2016)
La Facultad de Ingeniería nace en 1951 y la ingeniería en automatización 1975 (Universidad Autónoma de Querétaro, 2019) y línea terminal mecatrónica lograron la certificación internacional The Accreditation Board for Engineering and Technology (ABET) con la que se garantiza un programa de calidad a nivel mundial. (ABET, 2020).

Ingeniería en automatización durante los años 2015-2018 fue una población 149 egresados, información proporcionada por la Coordinación de seguimiento de egresados de la Facultad de Ingeniería, ( Salinas Valdez, 2019) representando $28 \%$ la línea terminal mecatrónica con 42 estudiantes de esta.

Gráfica 2. Ingeniería en automatización seguimiento de egresados 2015-2018.

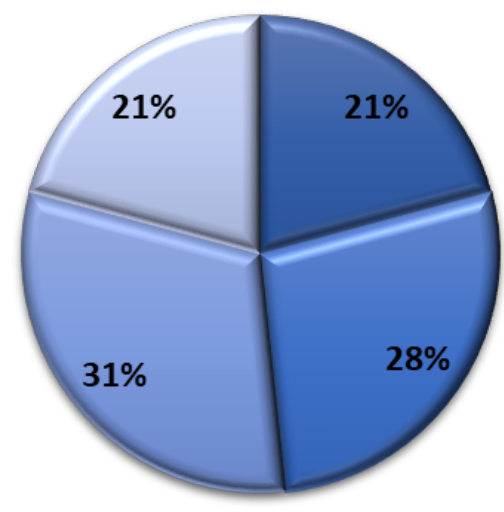

- Línea terminal en electrónica

- Línea terminal en mecatronica

L Línea terminal en instrumentación y control de procesos

a Línea terminal sistemas industriales

Fuente: Elaboración propia ( Salinas Valdez, 2019)

Los estudiantes activos 2015- 2018 en la gráfica 2 en su totalidad es de 196 estudiantes, distribuidos de la siguiente forma el 39\% son estudiantes de la línea terminal en electrónica en primer lugar, el segundo lugar la línea terminal mecatrónica con un 35\% que representa 69 estudiantes activos, en tercer lugar se tiene la línea terminal en instrumentación y control de procesos con un porcentaje del $27 \%$ y en cuarto lugar la línea terminal sistemas industriales que desaparece por la creación de la ingeniería industrial. Gráfica 2. 
Gráfica 2 Estudiantes activos 2015-2018
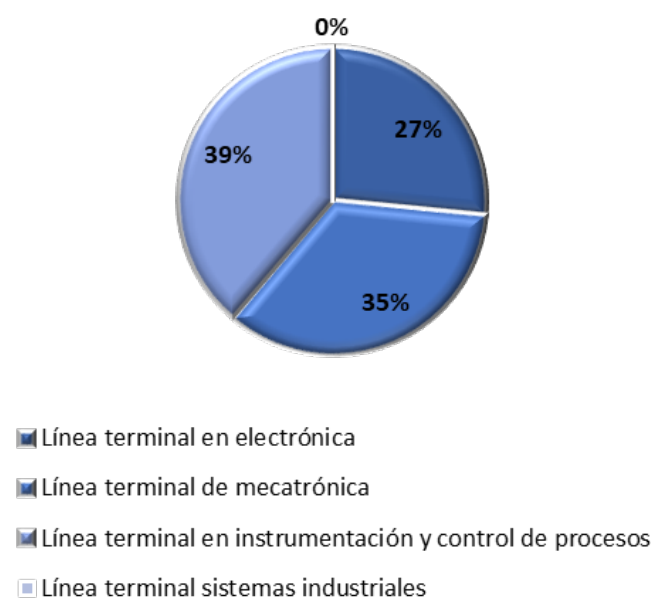

Fuente: Elaboración propia (Aveldaño Juárez , 2019)

La muestra que se considero fue la línea terminal de mecatrónica para ingenieros en automatización representada a forma de resumen grafica 3 , donde el número de estudiantes egresado 69 representado un $61 \%$ y estudiantes e $\mathbf{4 2}$ con un 39\%, considerando la población. Gráfica 3

Gráfica 3 Resumen de estudiantes activos y egresados 2015-2018

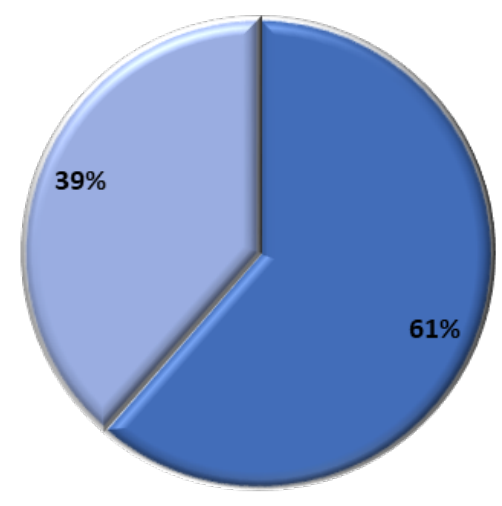

四 Activos 四Egresados

Fuente: Elaboración propia. (Aveldaño Juárez , 2019)

( Salinas Valdez, 2019)

\section{Resultados}

En el entorno fenomenológico y etnográfico de los estudiantes activos y egresados, se encuentran con un cambio de gobierno importante de derecha a izquierda, el emprendimiento lo aplican la corriente de derecha con un paternalismo hacia el emprendedor, prestando para sus proyectos a fondo perdido, esto no creo conciencia financiera. Los préstamos otorgados a los emprendedores terminaban en fracasar las empresas creadas.

Lo necesario era preparar a nuestros estudiantes en ámbitos emprendedores $\mathrm{y}$ financieramente en proyectos de tecnología con impacto social, para una toma decisiones con base reales, asumiendo sus derechos $y$ obligaciones.

Por tal motivo se realizó la indagación de que sucedía con estudiantes activos y egresados para su valoración, y presentar una solución real y buscando las mejores alternativas como es la educación, hoy con un gobierno de izquierda donde su perspectiva del emprendimiento es diferente hacia el impacto social, a los más vulnerables.

Se realizaron las encuestas en los estudiantes activos representando un $39 \%$ de la población y de los egresados representa un $28 \%$, esto fue porque al egresar el estudiante algunos datos que se tienen son cambiados y la localización es difícil. Gráfica 4.

Gráfica 4. Encuestados

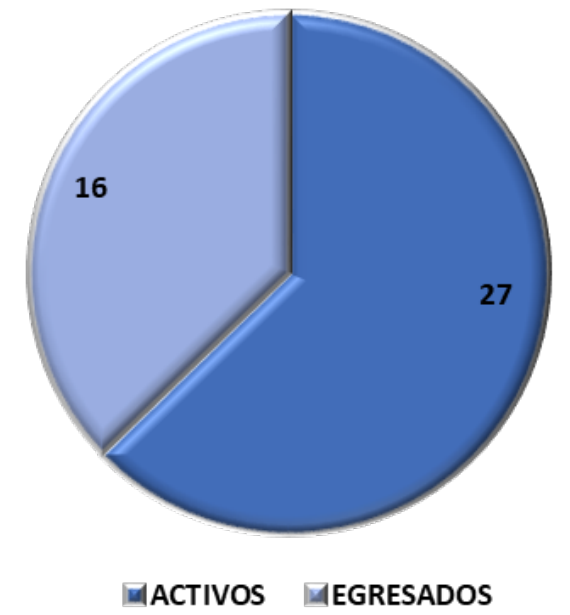

Fuente: Elaboración propia.

En la grafía 5 se ve reflejado que es una ingeniería de mayormente de hombres y minoría de mujeres de egresados con un 33\% y activos $17 \%$ de mujeres, sin embargo, los proyectos de emprendimiento de los activos tienen que ver 
con problemas que enfrentan las mujeres, por mencionar alguno el botón de pánico para cuando la mujer se sienta en situaciones vulnerables.

Gráfica 5. Encuestados género.

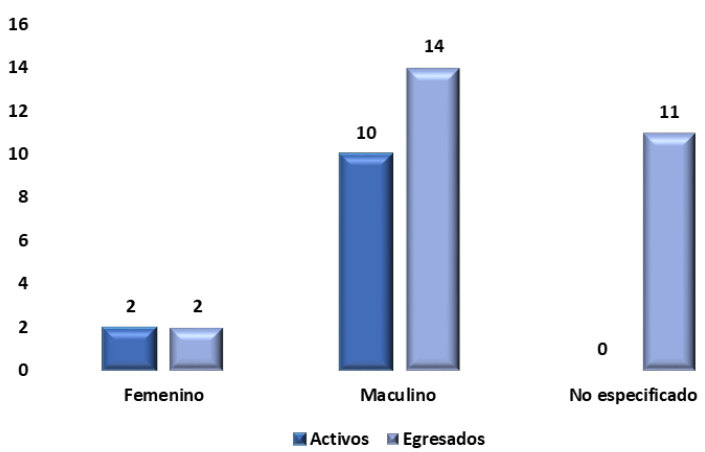

Fuente: Elaboración propia.

El estado civil gráfica 6 en el estudio fenomenológico de la investigación es de los estudiantes activos $89 \%$ son solteros, $4 \%$ casados y $7 \%$ otros, de los estudiantes egresados el $75 \%$ son solteros y un $25 \%$ en unión libre.

Gráfica 6 Encuestados estado civil.

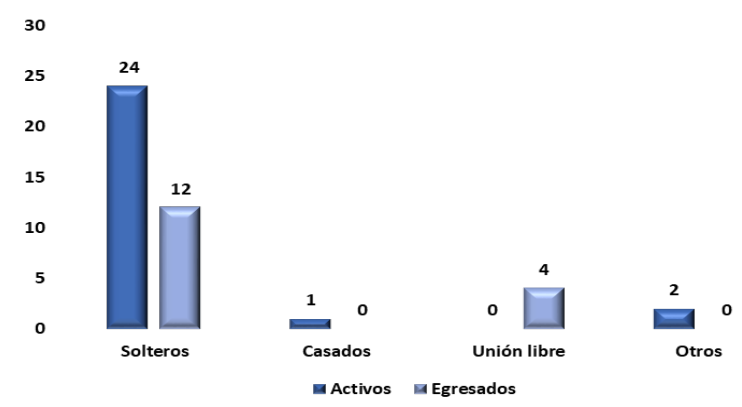

Fuente: Elaboración propia.

En la gráfica 7 de los estudiantes activos que participaron en la investigación el $37 \%$ son de 3 4 semestre, $26 \%$ son de los semestres 5 y 6 semestre, con un porcentaje $26 \%$ son de $7-8$ semestre, 9 a 10 semestre con un $7 \%$ y por último $4 \%$ de 1 a 2 semestre. La muestra de la investigación sumando los porcentajes de 3 a 8 semestre representa un $89 \%$, es decir que su formación en emprendimiento comienza en 3 semestre de la ingeniería.
Gráfica 7. Estudiantes activos.

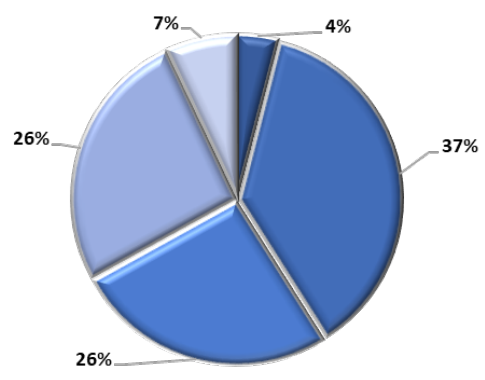

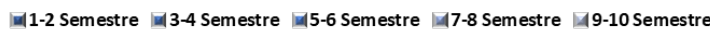

Fuente: Elaboración propia.

Gráfica 8 ¿Qué tipo de empresa tienes? Activos y Egresados

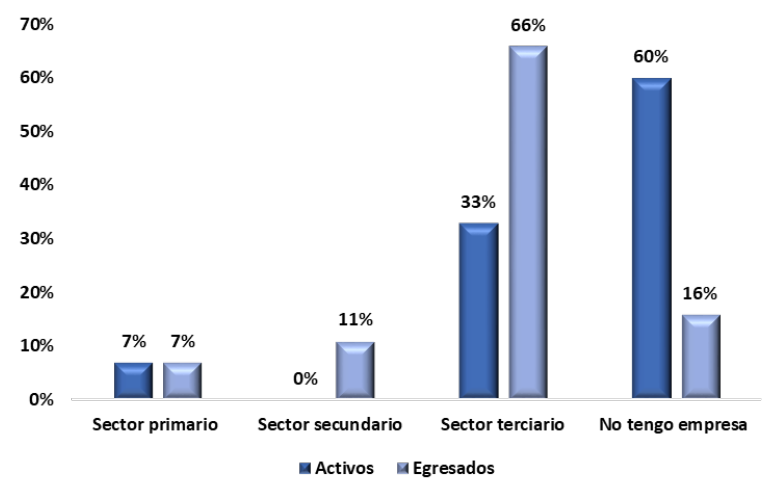

Fuente: Elaboración propia.

Gráfica 8 se muestra que los egresados buscan ser independientes y crear sus propias empresas representando $66 \%$ y correlacionado con los estudiantes activos que no cuentan con empresa con un $60 \%$ y el sector donde se interesan es los terciario, el sector primario con un 7\% tanto activos y egresados y el sector secundario con $11 \%$ es decir de transformación son de los egresados. Determinando que es necesario prepararlos en el emprendimiento. 
Gráfica 9 Los programas de estudio que cursaste fomentaron parte del emprendimiento.

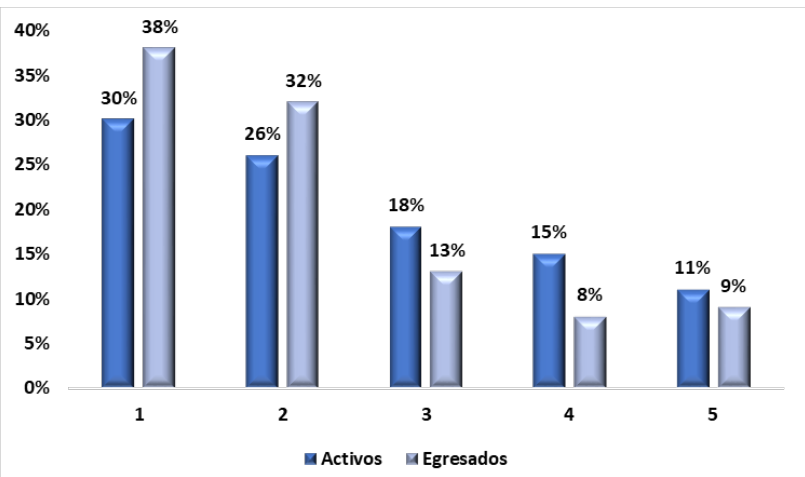

1 no me formaron y 5 me formaron. Egresados y estudiantes activos

Fuente: Elaboración propia.

Las asignaturas de emprendimiento como se comentó al inicio del documento son optativas no son materias curriculares, es decir, no todos llevan estas materias, el estudiante decide cual llevar. En la gráfica 9 de los activos están entre el 4 al 5 sumando los porcentajes es $26 \%$ y egresados $17 \%$ donde los egresados fueron formados en emprendimiento, estudiando los rangos 1 y 2 en activos representa $56 \%$ y egresados $70 \%$ no recibieron formación emprendedora, los del rango 3 son los indecisos que no saben que es el emprendimiento como lo mencionaron en la encuesta estos representan $18 \%$ en los activos y 13\% egresados.

Gráfica 10 Las asignaturas que te ayudaron a formarte como emprendedor fueron: Estudiantes activos y egresados.

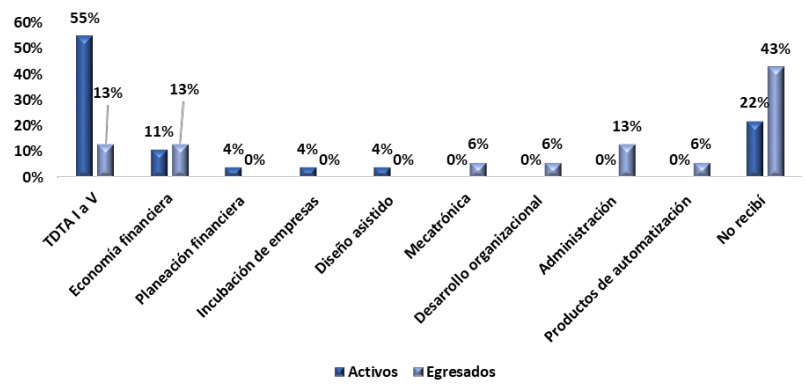

Fuente: Elaboración propia.
En los inicios de esta investigación se menciona que las materias de emprendimiento se encuentran en optativas y no curriculares por tal motivo existe una variedad de materias que no todos reciben, por eso los porcentajes $22 \%$ y $43 \%$ de estudiantes activos y egresados Gráfica 10 donde especifican que no han recibido las asignaturas que le ayudan a formar como emprendedores, la materia de Taller de Desarrollo de Tecnología en Automatización (TDTA) con un 55\% activos y 13\% en egresados, esta materia una materia obligatoria depende del materia que imparte la materia agrega o no algunos temas de emprendimiento dependiendo de la formación del docente que imparte la materia. Y las demás materias son optativas.

Gráfica 11 ¿Cuál fue tu primera empresa? Estudiantes activos y egresados.

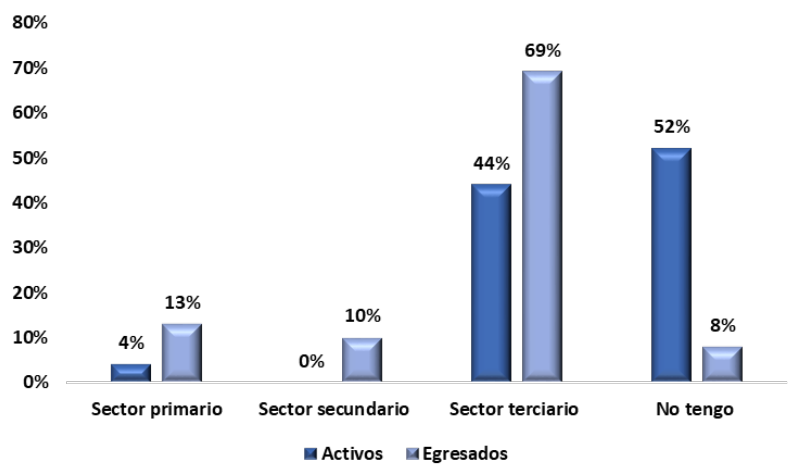

Fuente: Elaboración propia.

En las gráficas 11 Los estudiantes su primera empresa en los activos son del sector terciario con 44\%, al igual delos egresados $69 \%$ y no tienen empresa $52 \%$ y $8 \%$ de activos $y$ egresados, si se relaciona la gráfica 8 ¿Qué tipo de empresa tienes? Activos y egresados donde coincide en que la empresa que emprendieron fue $33 \%$ y $56 \%$ activos y egresados existe una correlación especificando que el sector donde se implemento fue el terciario, en los no tienen empresa $60 \%$ y 16\% estudiantes activos y egresados respectivamente. 
Gráfica 12 Has participado en algún evento de emprendimiento. Estudiantes activos y egresados

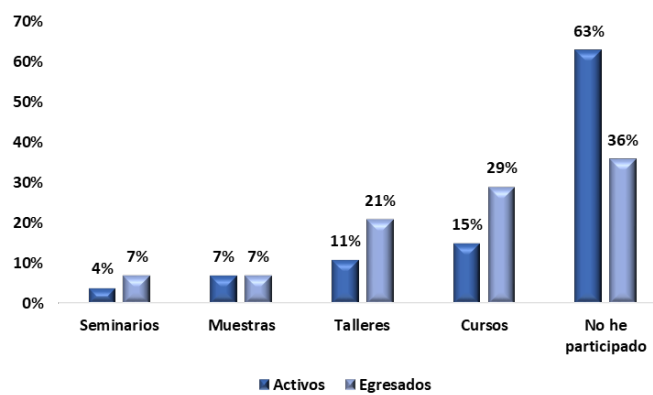

Fuente: Elaboración propia.

Al no contar todos con el aprendizaje del emprendimiento, se ven en la necesidad de contratar estos por fuera de su formación académica se percibe en la gráfica 12 Has participado en algún evento de emprendimiento. De los estudiantes activos con $63 \%$ y de egresados $36 \%$ no participaron en estos cursos, comentando algunos que al formar estas empresas han cometido errores por desconocimiento que no exime como dice la autoridad de las sanciones fiscales. Hoy es obligatorio el enseñar emprendimiento y finanzas establecido por la Ley generar de educación mencionado anteriormente entrada en vigor 30 de septiembre de 2019. (Cámara de diputados del H. Congreso de la Unión, 2019)

Imagen 2 Plan de estudio de Ingeniería Materia Básicas

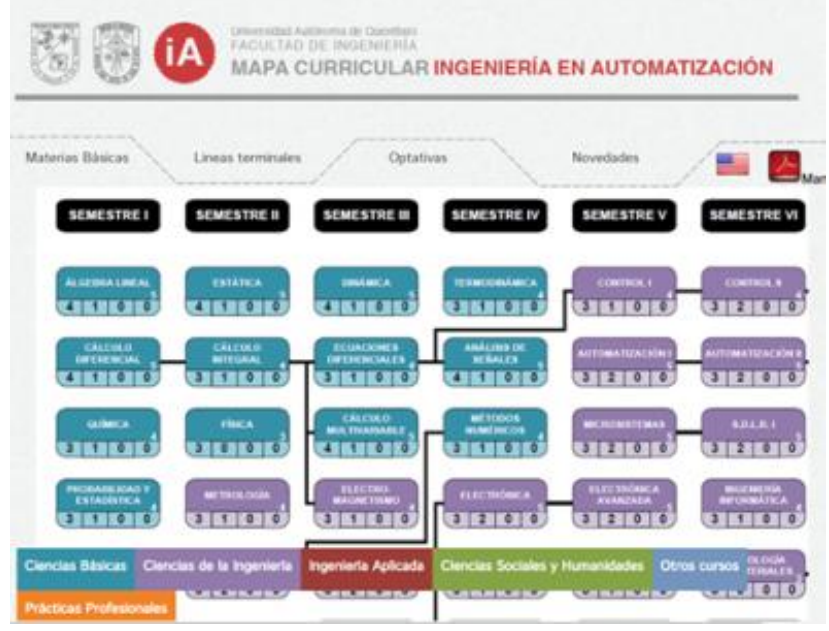

Fuente: (Universidad Autónoma de Querétaro, 2019)
Imagen 3 Plan de estudio de Ingeniería Materia Básicas 2

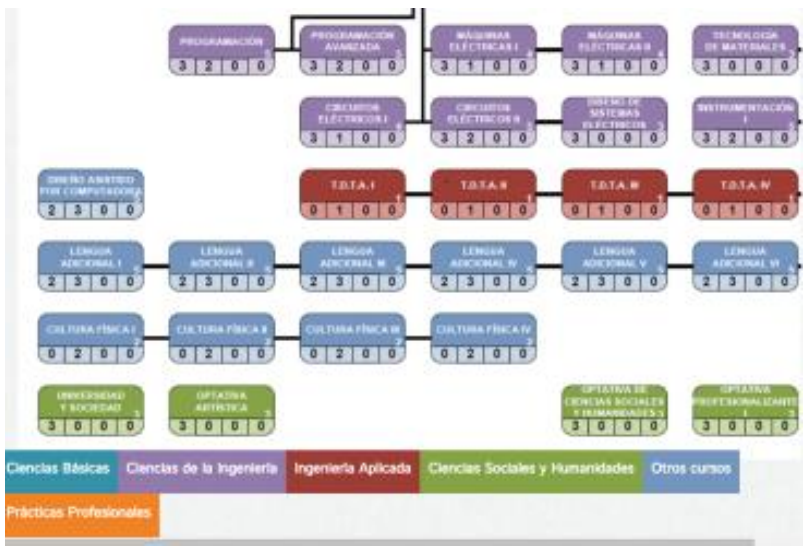

Fuente: (Universidad Autónoma de Querétaro, 2019)

Imagen 4 Línea terminal Mecatrónica

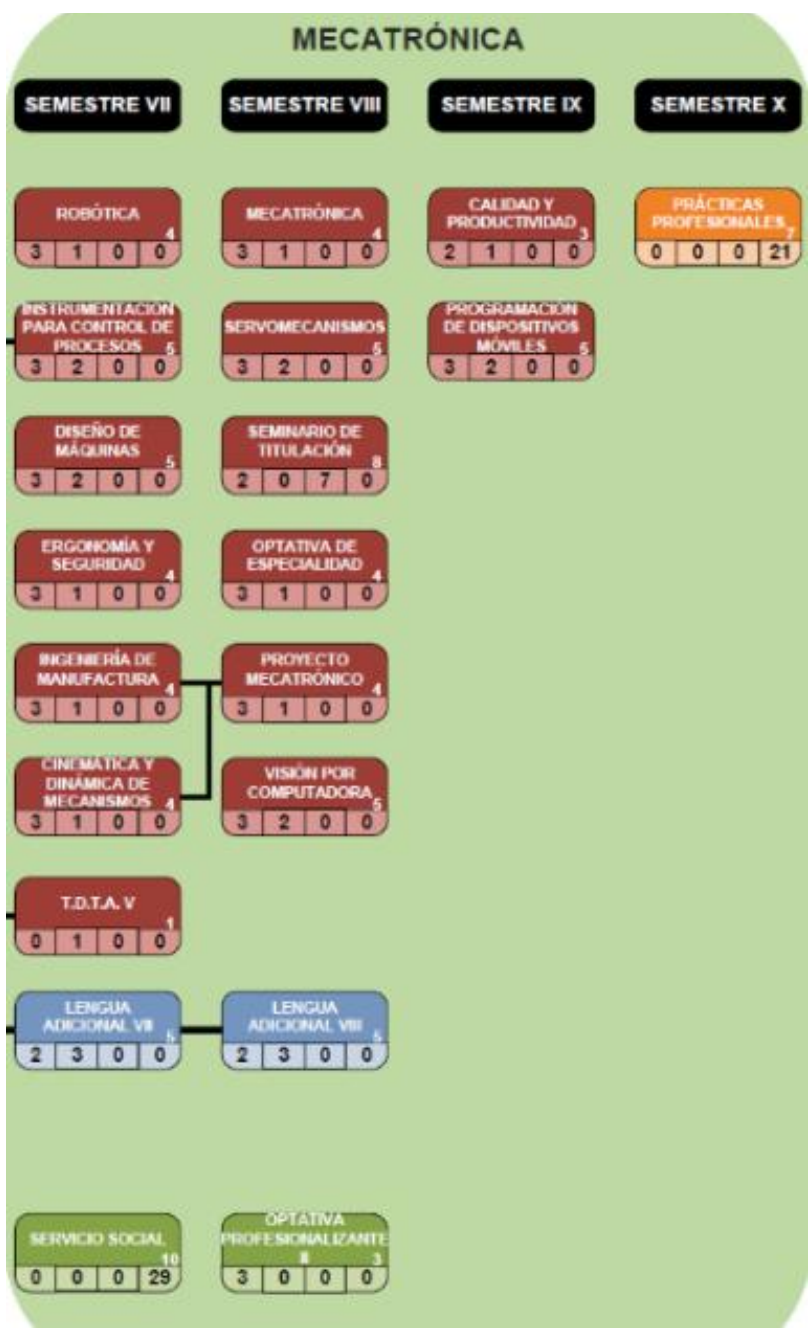

Fuente: (Universidad Autónoma de Querétaro, 2019) 
Imagen 6 Taller de Desarrollo de Tecnología en Automatización.

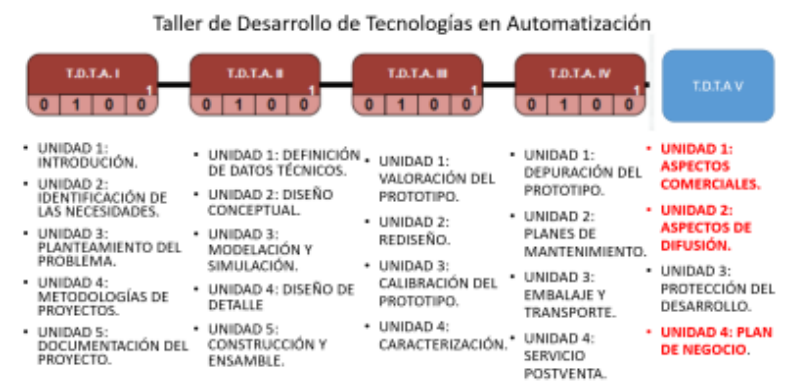

Fuente: Elaboración propia.

En la imagen 2 y 3 se observa que no existe alguna materia de emprendimiento en su formación básica, en el área de mecatrónica su formación en el Taller de Desarrollo de Tecnología en Automatización se observa en T.D.T.A V Imagen 4, 5 y 6 aspectos de comercialización, cuando antes de realizar una proyecto primero se necesita realizar un estudio de mercado para establecer su nivel de comercialización, es decir, se comienza al revés primero se hace el proyecto de acuerdo a las necesidades de los investigadores y no a las necesidades de la industria, cuando se debe empatar con la industria realizado contratos con necesidades reales antes de comenzar un proyecto.

Lograr un liderazgo en los jóvenes estudiantes que son capaces de comercializar su producto, y que no solo sirvan para pasar la materia o para crear productividad al investigador, quizá en conjunto se puede fomentar el ecosistema emprendedor de innovación y emprendimiento en ambientes universitarios para lograr un impacto social.

Imagen 7 Optativas Profesionalizantes I

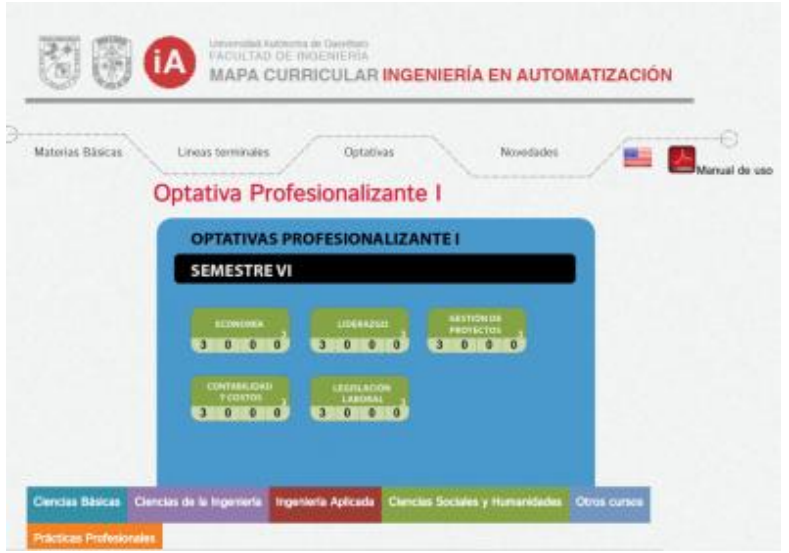

Fuente: (Universidad Autónoma de Querétaro, 2019)
Imagen 8 Optativas Profesionalizantes II

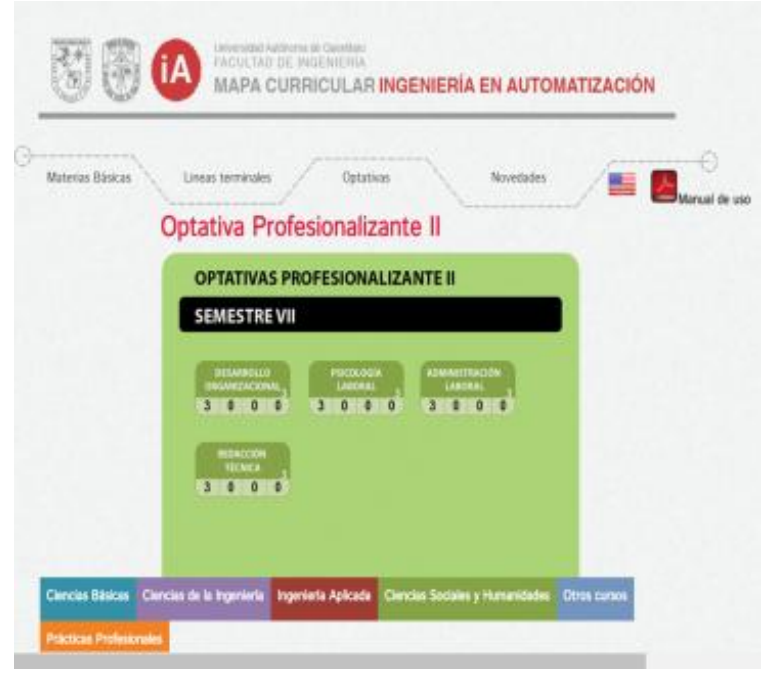

Fuente: (Universidad Autónoma de Querétaro, 2019)

En las imágenes 7 y 8 se muestran optativas donde el estudiante decide cual quiere cursar, y no tienen una programación continua son aisladas sin tener un producto final. Si quieren tener estudiantes lideres emprendedores, deben trabajar en materias curriculares y continuas donde le permitan al estudiante formarse en el emprendimiento.

\section{Conclusión}

En esta investigación se indago las alternativas para los estudiantes activos cuenten con el aprendizaje del emprendimiento tecnológico e innovación desde un ambiente universitario con impacto a la sociedad, ya propuesto por la Ley general de educación (Cámara de diputados del H. Congreso de la Unión, 2019), es decir, que la ingeniería en automatización línea terminal en mecatrónica Facultad de ingeniería en Universidad Autónoma de Querétaro se proyectó la necesidad por medio de las asignaturas llamadas optativas no cumpliendo en su totalidad en la aplicación del emprendimiento y finanzas como materia curricular, los resultados de esta investigación confirman el cambio de la ley, dando respuesta a:

¿Estableciendo un programa de emprendimiento tecnológico e innovación, cumplirá con la LGE? 
La Secretaria de Educación por medio de la Ley General de Educación agrega el 30 de septiembre del 2019 en su artículo 30 en su fracción:

...Artículo 30. Los contenidos de los planes y programas de estudio de la educación que impartan el Estado, sus organismos descentralizados y los particulares con autorización o con reconocimiento de validez oficial de estudios, de acuerdo al tipo y nivel educativo, serán, entre otros, los siguientes... XIV. La promoción del emprendimiento, el fomento de la cultura del ahorro y la educación financiera... (Cámara de diputados del $\mathrm{H}$. Congreso de la Unión, 2019)

La Ley general de educación (Cámara de diputados del H. Congreso de la Unión, 2019)obliga a las instituciones de educación la aplicación de la investigación, la ciencia, tecnología y la innovación como la comprensión, aplicación y el uso responsable de la misma, como se menciona enseguida:

Artículo 30. Los contenidos de los planes y programas de estudio de la educación que impartan el Estado, sus organismos descentralizados y los particulares con autorización o con reconocimiento de validez oficial de estudios, de acuerdo al tipo y nivel educativo, serán, entre otros, los siguientes ... IV. El fomento de la investigación, la ciencia, la tecnología y la innovación, así como su comprensión, aplicación y uso responsables... (Cámara de diputados del H. Congreso de la Unión, 2019)

El objetivo general realizar un estudio del impacto del emprendimiento recibido durante su formación como ingenieros en automatización línea terminal mecatrónica de estudiantes activos y egresados 2015-2018, esto permitirá desarrollar un programa de emprendimiento.

Se menciona que las materias de emprendimiento se encuentran en optativas y no curriculares por tal motivo existe una variedad de materias que no todos reciben, por eso los porcentajes $22 \%$ y $43 \%$ de estudiantes activos y egresados donde especifican que no han recibido las asignaturas que le ayudan formarse como emprendedores, la materia de Taller de Desarrollo de Tecnología en Automatización
(TDTA) con un 55\% activos y 13\% en egresados, esta materia una materia obligatoria, los rubros de esta están diseñado por ingenieros y son en los últimos TDTA donde les hablan de comercialización, cuando debería de ser desde los primeros semestres para que el proyecto tenga una viabilidad financiera y no en los últimos semestres, cuando el estudiante se da cuenta que no es viable. Gráfica 10

Metodología es cuantitativa, fenomenológica y etnográfica, en la Universidad Autónoma de Querétaro en la Facultad de Ingeniería línea terminal mecatrónica de activos y egresados del 2015 al 2018, por medio de las encuestas. Gráfica 4 Se realizaron las encuestas en los estudiantes activos representando un 39\% de la población y de los egresados representa un $28 \%$, esto fue porque algunos datos que se tienen han cambiado y la localización es difícil de los egresados. Es fenomenológica se realizó un estudio del entorno donde se desarrolló la investigación económica y política y etnográfica en el Cerro de las campanas S/N, colonia centro en Querétaro, donde esta centro universitario de la Universidad Autónoma de Querétaro.

Hipótesis cierta si se realiza un estudio del aprendizaje emprendimiento tecnológico e innovación desde un ambiente universitario con impacto social, recibido durante su formación como ingenieros en automatización línea terminal mecatrónica de estudiantes activos y egresados 2015-2018, entonces se logrará desarrollar un programa de estudio de emprendimiento.

Recomendaciones en los planes de estudio se contemple estas asignaturas debido a la importancia al salir de la carrera sin una orientación multidisciplinaria y prepararlos para la vida real de competencia y disciplina en el ecosistema emprendedor innovador en ambientes universitario.

Prepararlos para proponer proyectos integradores donde el estudiante se prepare en un ecosistema emprendedor y pierda el miedo, encontrando seguridad de presentar sus proyectos que son únicos, demostrando ser capaz de resolver cualquier situación que se presente y prepararlo para la toma de decisiones.

Se establece un programa de emprendimiento Imagen 10 -17, aprobado en la Coordinación de 
emprendimiento e incubadora de empresas UAQ, donde los jóvenes pueden desarrollar esta parte del ecosistema emprendedor innovador en ambientes universitarios y tener un impacto social en un mundo real.

Imagen 10 programa propuesto 1

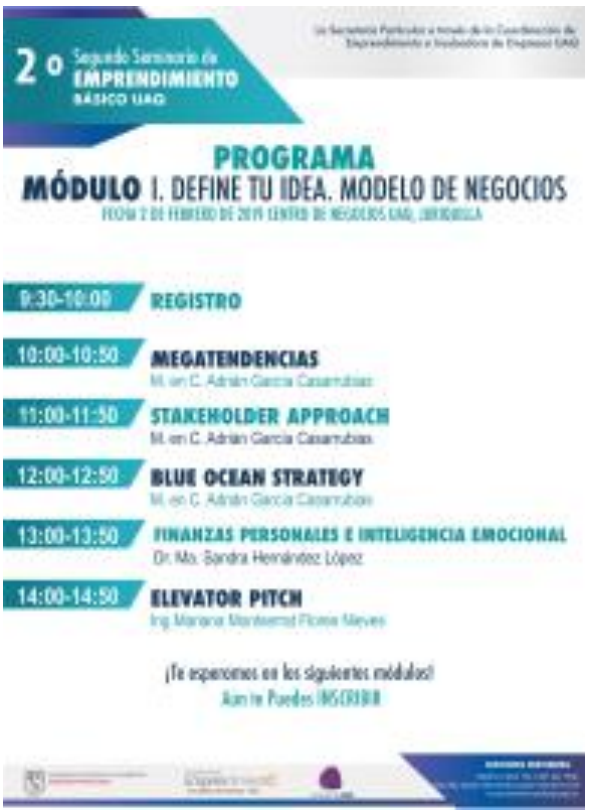

Fuente: Elaboración propia.

Imagen 11 programa propuesto 2

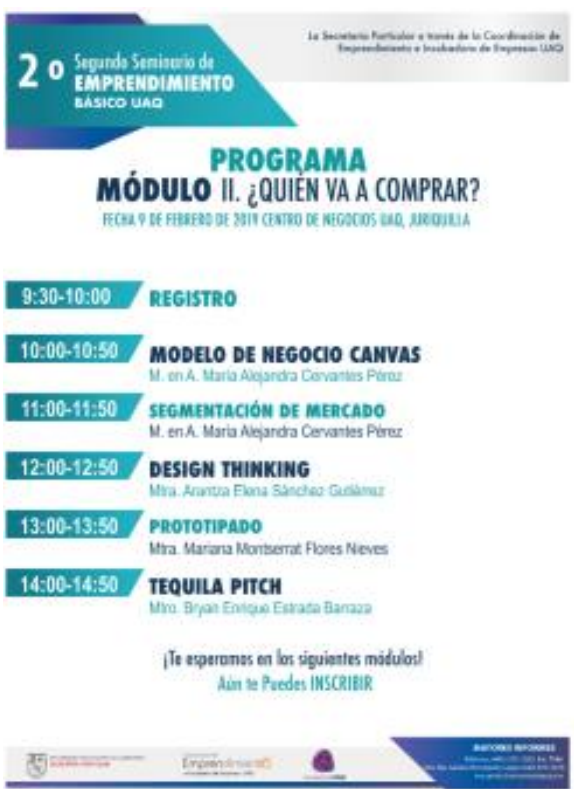

Fuente: Elaboración propia.
Imagen 12 programa propuesto 3

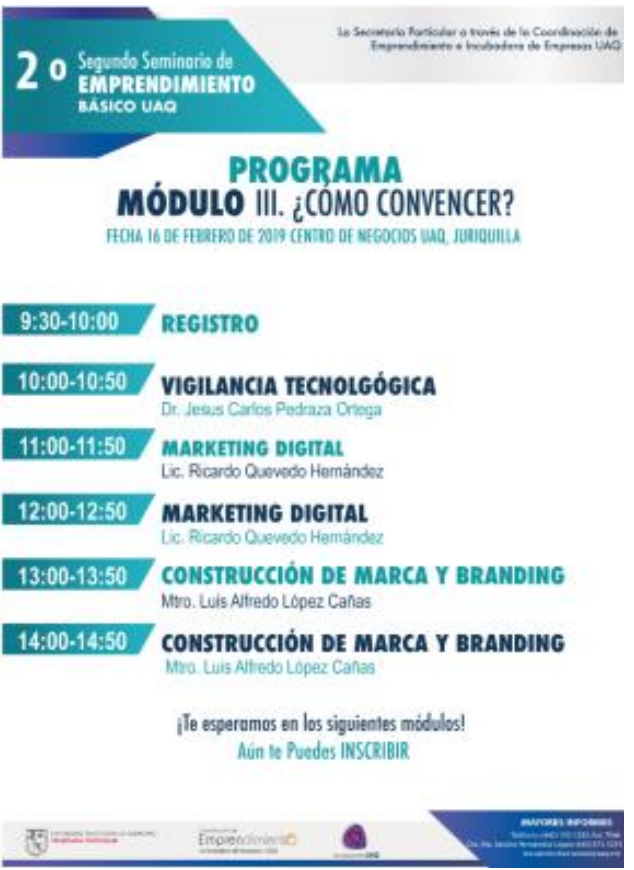

Fuente: Elaboración propia.

Imagen 13 programa propuesto 4

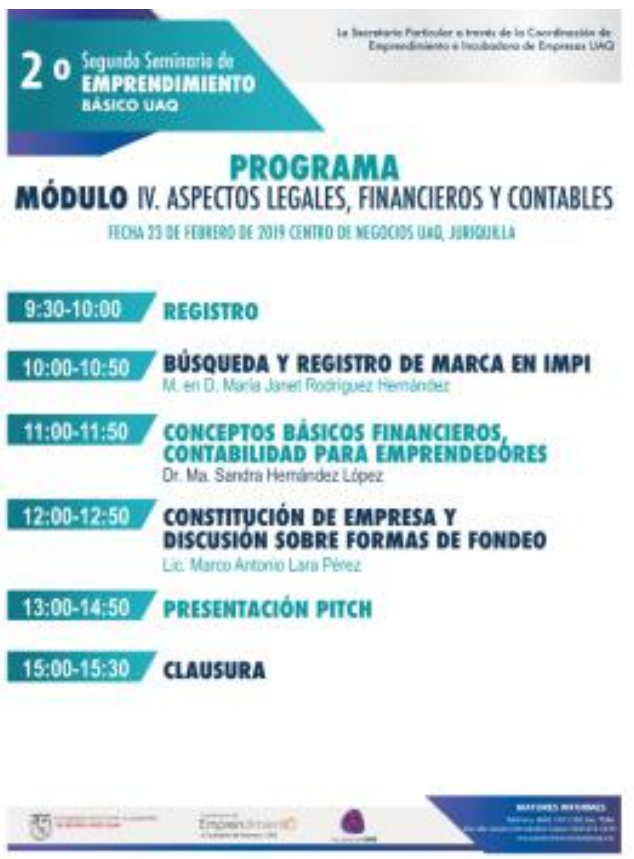

Fuente: Elaboración propia. 
Imagen 14 programa propuesto 5

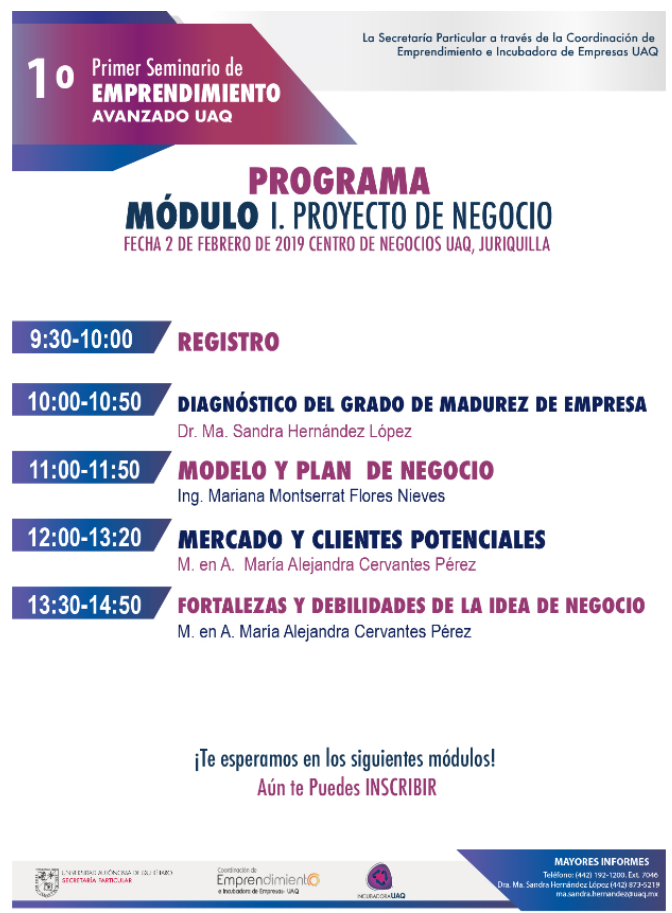

Fuente: Elaboración propia.

Imagen 15 programa propuesto

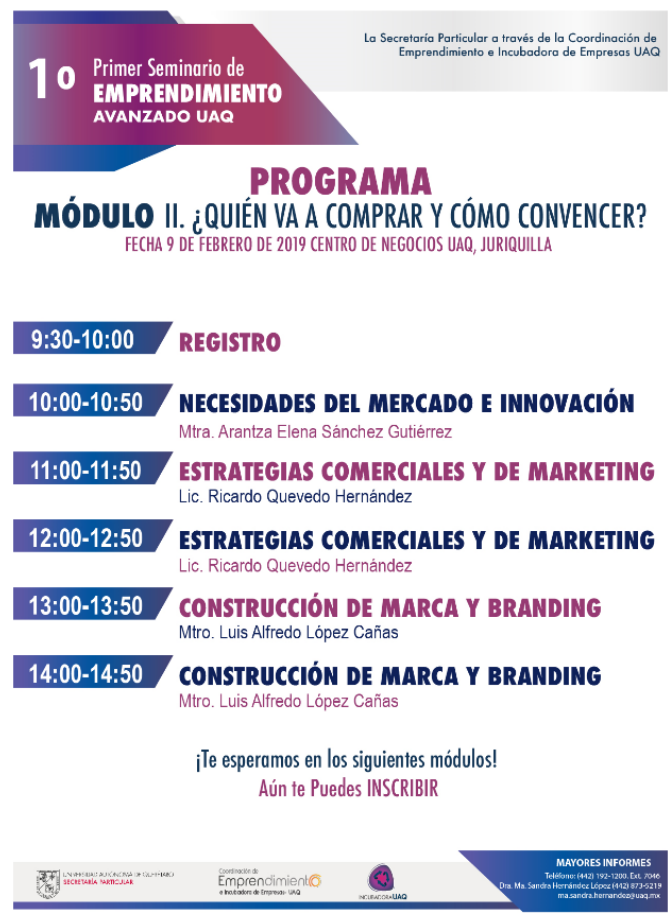

Fuente: Elaboración propia.
Imagen 16 programa propuesto 7

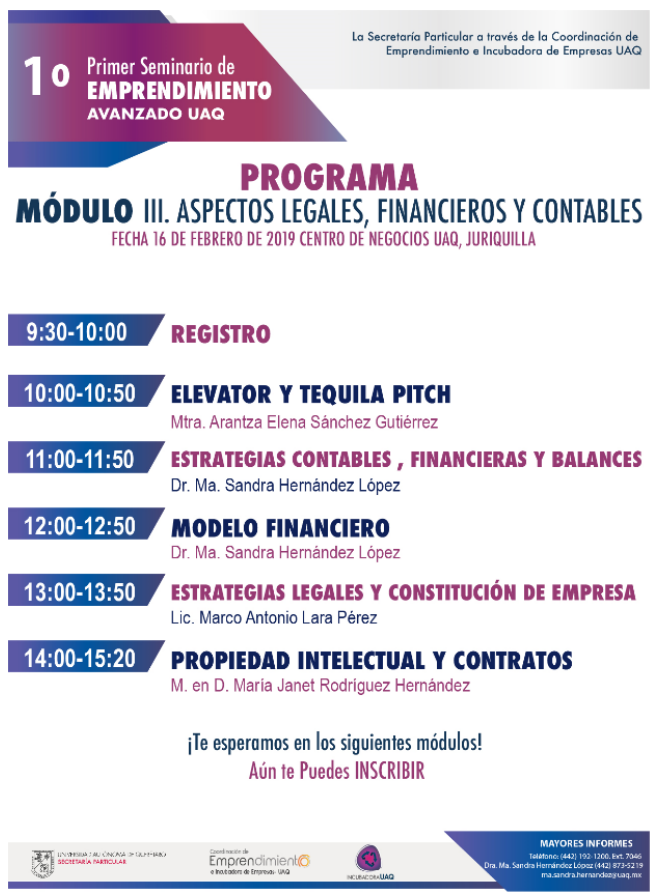

Fuente: Elaboración propia.

Imagen 17 programa propuesto 8

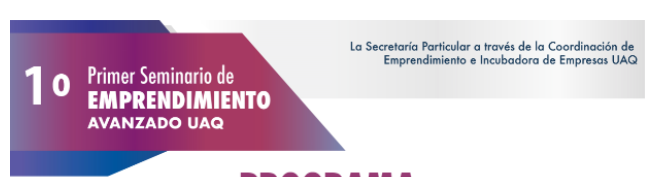

PROGRAMA

MÓDULO IV. EQUIPO Y LIDERAZGO EMPRENDEDOR FECHA 23 DE FEBRERO DE 2019 CENTro DE NEGOCOOS UAQ, JURIQUULLLA

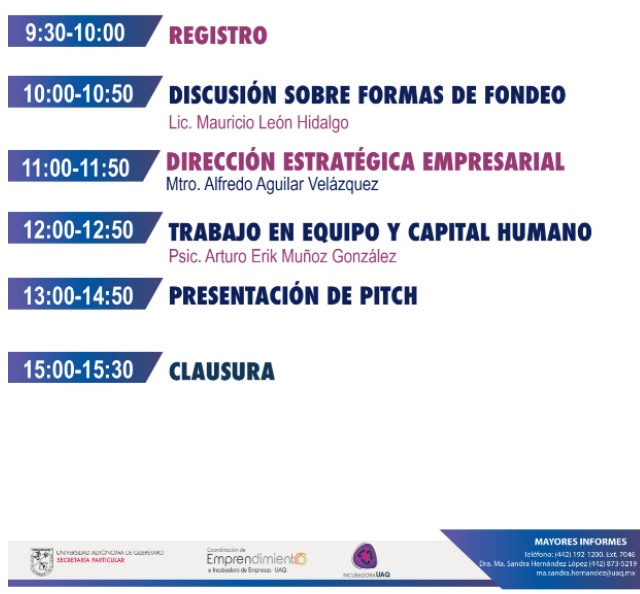

Fuente: Elaboración propia. 
Afirman que es indispensable contar con estas asignaturas en la gráfica 8 se muestra que los egresados buscan ser independientes y crear sus propias empresas representando $66 \%$ y correlacionado con los estudiantes activos que no cuentan con empresa con un $60 \%$ y el sector donde se interesan es los terciario, el sector primario con un $7 \%$ tanto activos y egresados y el sector secundario con $11 \%$ es decir de transformación son de los egresados. Determinando que es necesario prepararlos en el emprendimiento.

La necesidad de las asignaturas de emprendimiento establecidas al inicio del documento es optativa no son asignaturas curriculares, es decir, no todos llevan estas asignaturas, el estudiante decide cual llevar. En la gráfica 9 de los activos están entre el 4 al 5 sumando los porcentajes es $26 \%$ y egresados $17 \%$ donde los egresados fueron formados en emprendimiento, estudiando los rangos 1 y 2 en activos representa $56 \%$ y egresados $70 \%$ no recibieron formación emprendedora, los del rango 3 son los indecisos que no saben que es el emprendimiento como lo mencionaron en la encuesta estos representan 18\% en los activos y $13 \%$ egresados.

Es importante destacar que la Facultad de ingeniería en ingeniería en automatización busca estar a la vanguardia, sé que necesitan trabajar en todas las carreras universitarias y no solo en una, esto permitirá que sean ejemplo para otras, y puedan cumplir con los lineamientos que las autoridades de educación requieran. 


\section{Referencias}

Alvarado Muñoz, O., \& Rivera Martínez, W. F. (2011). Universidad y emprendimiento, aportes para la formación de profesionales emprendedores. Cuadernos de Administración, 27 (45), 61-74.

Hernández Rodríguez, C., \& Arano Chávez, R. M. (2015). El desarrollo de la cultura emprendedora en estudiantes universitarios para el fortalecimiento de la visión empresarial. Ciencia Administrativa, 32, 30.

Mendieta-Izquierdo, G., Ramírez-Rodríguez, J. C., \& Fuerte, J. A. (2015). La fenomenología desde la perspectiva hermenéutica de Heidegger: una propuesta metodológica para la salud pública. Rev. Fac. Nac. Salud Pública, 435-443.

Salinas Valdez, P. (2019, Abril 8). Seguimiento de egresados. Coordinador de seguimiento de egresados Facultad de Ingeniería de la Universidad Autónoma de Querétaro. (S. Hernández López, Interviewer)

Sparano Rada, H. (2014). Emprendimiento en américa latina y su impacto en la gestión de proyectos. Revista Dimensión Empresarial, 12 (2), 95.

Vásquez, C. (2017). Educación para el emprendimiento en la universidad. Estudios de la Gestión: revista internacional de administración (2), 121-147.

Universidad Autónoma de Querétaro. (2019). Facultad de Ingeniería . Retrieved from https://www.uaq.mx/index.php/oferta-educativa/programas-educativos/fi/licencuaturasfi/ingenieria-en-automatizacion

Universidad Autónoma de Querétaro. (2019, Abril 4). Facultad de Ingenieria . Retrieved from Ingenniería en

Automatización

http://ingenieria.uaq.mx/web/files/educacion/eduLicen/ingAuto/automatizacionPC/index.html

Aveldaño Juárez, J. L. (2019, Abril 14). Estudiantes activos de automatización. Coordinador de la Carrera de Ingeniería en automatización . (S. Hernández López, Interviewer)

ABET. (2020). Retrieved from http://www.abet.org

Álvarez Perdomo, P. E., Andrade Alcívar, M. A., Bravo Salvatierra, J. X., Rodríguez Martínez, M. E., \& Govea Vilcacundo, J. L. (2016). La innovación y el emprendimiento: necesidades en la educación superior. Didáctica y Educación., 219-246.

Aponte Figueroa, G. M. (2015). El proceso de gestión de innovación tecnológica: sus etapas e indicadores relacionados. Revista Venezolana de Análisis de, XXI ( 1 ), 59.

Arredondo Trapero, F., Vázquez Parra, J. C., \& de la Garza, J. (2015). Factores de innovación para la competitividad en la Alianza del Pacífico. Una aproximación desde el Foro Económico Mundial. Estudios Gerenciales, 301.

Becerra Rodríguez, F., Serna Gómez, H. M., \& Naranjo Valencia, J. C. (2013). Redes empresariales locales, investigación y desarrollo e innovación en la empresa. Cluster de herramientas de Caldas, Colombia. Estudios Gerenciales, 29 (127), 249.

Cavero Clerencia, J. M., \& Ruíz Clerencia, D. (2017). Educación para la innovación y el emprendimiento una educación para el futuro. 11-199.

Cámara de diputados del H. Congreso de la Unión. (2019). Ley General de Educación. Retrieved from http://www.diputados.gob.mx/LeyesBiblio/index.htm

Cienfuegos Velasco, M., \& Cienfuegos Velasco, A. (2016). Lo cuantitativo y cualitativo en la investigación. Un apoyo a su enseñanza. Revista Iberoamericana para la Investigación y el Desarrollo Educativo, 7 (13).

Escobar Londoño, J. V., Castaño, D. A., Ruiz Ruiz, M. P., \& Restrepo Botero, J. C. (2016). Evaluación auténtica del impacto social de procesos, proyectos y productos de investigación universitaria: un acercamiento desde los grupos de investigación. La revista lasallista de investigación, 13 (1), 169.

Del Canto, E., \& Silva Silva, A. (2013). Metodología cuantitativa: abordaje desde la complementariedad en ciencias. Revista de Ciencias Sociales, 25-34.

Gutiérrez Giraldo, M. C., Agudelo Cely, N., \& Orlando Caro, E. (2016). La etnografía educativa virtual y la formación docente. Praxis \& saber, 41-62. 
Gutiérrez Olvera, S. (2015). Emprendimiento en las empresas familiares. Revista Iberoamericana de Contaduría, Economía y Administración, 4 (7), 3.

García Gajardo, F., Fonseca Grandón, G., \& Concha Gfell, L. (2015). Aprendizaje y rendimiento académico en educación superior: un estudio comparado. Revista Electrónica "Actualidades Investigativas en Educación", 15 (3), 2.

INADEM. (2018). Instituto Nacional del Emprendedor. Retrieved Enero 6, 2019, from https://www.inadem.gob.mx/

INEGI. (2019, Diciembre 24). Instituto nacional de estadística y geografía. Retrieved Enero 6, 2020, from https://www.inegi.org.mx/sistemas/bie/

Hernandes, A. C., Baffa Lourenço, A., Colombo Junior, P. D., Barsi Andreeta, M. R., \& Guillarón, J. J. (2014). Actividad de Investigación Científica en ambiente universitario: un estudio de sus contribuciones para estudiantes de la Enseñanza Media. 29.

Jorquera, F., Marillanca, A., \& Loyola, K. (2015). Salud mental e ingreso al ambiente universitario: una aproximación a los significados de estudiantes de primer año de la Pontificia Universidad Católica de Valparaíso. Retrieved Marzo 29, 2019, from http://opac.pucv.cl/pucv_txt/txt1500/UCD1825_01.pdf

Lizano Barrantes, C., Arias Mora, F., Cordero García, E., \& Ortiz Ureña, A. (2015). Relación entre estilo de aprendizaje y rendimiento académico en estudiantes de farmacia de la Universidad de Costa Rica. Revista Digital de Investigación en Docencia Universitaria, 49.

Martín Carbajal, M., \& Gutiérrez Hernández, R. (2018). Diagnóstico y evaluación de las incubadoras de empresas de tecnología intermedia en Morelia, Michoacán. Economía y sociedad (39), 36 y 61.

Martinic, S. (2015). El tiempo y el aprendizaje escolar la experiencia de la extensión. Revista Brasileira de Educação, 20 (61), 485.

Mendoza León, J. G., \& Valenzuela Valenzuela, A. (2014). Aprendizaje, innovación y gestión tecnológica en la pequeña empresa Un estudio de las industrias metalmecánica y de tecnologías de información en Sonora. Contaduría y Administración, 257.

Morales Ramírez, D., \& Roux Rodríguez, R. (2015). Estudio de impacto social: antecedentes y línea base para San Fernando, Tamaulipas. Revista Internacional de Ciencias, XXV (1), 112.

Observatorio Estratégico de la Alianza del Pacífico. (2018). Brechas para el Emprendimiento en la Alianza del Pacífico. Colombia: Observatorio Estratégico de la Alianza del Pacífico.

OECD. (2018). The Measurement of Scientific, Technological and Innovation Activities. (Eurostat, Producer, \& $\quad$ OECD) Retrieved from Oslo https://icono.fecyt.es/sites/default/files/filepublicaciones/manualoslo2018.pdf

Orozco Idárraga, A. (2014). Impacto social y académico del programa interacción social y desarrollo ciudadano en tres localidades del departamento del atlántico. Psicogente, 478.

Ramírez Urquidy, M., Bernal, M., \& Fuentes, R. (2013). Emprendimiento y desarrollo manufactureros en las entidades federativas de México. Revista Problemas del Desarrollo, 169.

Rodríguez Restrepo, V. J., \& Larrota Castro, S. Y. (2014). El emprendimiento social de los estudiantes del programa de Administración de Empresas de la Universidad de la Salle. Sondeo de interés hacia las comunidades marginales de Bogotá. Punto de Vista, 5 (8), 113-131.

Roldán Vargas, 0. (2014). Incursión de los y las jóvenes en el mundo Universitario: tensiones entre ser y permanecer. Educ. Soc., Campinas, 35 (126), 143.

Saldarriaga Salaza, M. E., \& Guzmán González, M. F. (2018). Enseñanza del emprendimiento en la educación superior: ¿Metodología o modelo? EAN, 85, 125-142.

Sánchez García, J. C., Brizeida Hernández, A. W., \& Lizette Florez, J. (2017). Educación emprendedora: Estado del arte. Propósito y representaciones, 5 (2), 412. 